\title{
Traditional Bone-Setters and Fracture Care in Ekiti State, Nigeria
}

Owoseni Joseph Sina ${ }^{1,{ }^{*}}$ and Ibikunle Ayodele $\mathbf{M}^{2}$

${ }^{1}$ College of Medicine and Health Sciences (Medical Sociology), Afe Babalola University, Ado-Ekiti, Nigeria

${ }^{2}$ Department of Sociology, Ekiti State University Ado Ekiti, Nigeria

*Corresponding author: Owoseni Joseph Sina, College of Medicine and Health Sciences (Medical Sociology), Afe Babalola University, Ado-Ekiti, Nigeria, Tel: +2348066504953; E-mail: owoshynah@yahoo.com

Rec date: Jul 23, 2014; Acc date: Dec 31, 2014; Pub date: Jan 03, 2015

Copyright: ( 2015 Sina OJ, et al. This is an open-access article distributed under the terms of the Creative Commons Attribution License, which permits unrestricted use, distribution, and reproduction in any medium, provided the original author and source are credited.

\begin{abstract}
In Nigeria, the practice of traditional bone setting (TBS) is extensive as in other developing nations. Traditional bonesetters play an imperative role in fracture care. Though, the outcome of the intervention of TBS treatment is usually poor with profound effects on the patient in the future. This study seeks to examine the activities of traditional bone setters on fracture care in Ekiti State, Nigeria.
\end{abstract}

Survey method was applied in the study. 250 questionnaires were administered to owners of traditional bone centres and their clients respectively. Ten (10) of such centres were selected for the study in Ekiti.

The findings indicated that majority of the bone setters had little or no formal education. Many of them acquired the schema used through ancestral/supernatural power. The TBS lack basic knowledge of investigations and wound care while most of their clients patronized them because of poverty, ignorance and superstitious beliefs.

Provision of adequate basic equipment in health institutions may reduce the rate at which people utilize Traditional bone centres against medical advice.

Keywords: Traditional bone setters; Fracture; Care; Nigeria

\section{Introduction}

In Nigeria, with high level of accidents recorded every day, the traditional care givers still remain popular despite the high level of education and the existence of modern health care facilities [1]. In Nigeria, there are traditional medical practitioners - herbalists, bonesetters and religious practitioners. In the main, traditional healers and bonesetters were practicing long before orthodox medicine was introduced to the developing world Hoff, 1997.

Contemporarily, both orthodox and traditional medicines are coexisting side by side and both are patronized by patients [2]. Basically, in Nigeria, the traditional bone setters enjoy more confidence and patronage from people than any other group of traditional care-givers [3].

On the whole, the traditional bonesetter's practice is a highly specialized form of traditional medicine. It is usually passed from father to son but some outsiders also receive their training through apprenticeship [4]. Traditional bone setting is an old practice found almost in all communities of Nigeria especially in the rural communities of Ekiti State. Traditional Bone setting (TBS) is popular in Africa because its practitioners lay claims to supernatural influences [5].

World Health Organization (2002) describes traditional bonesetting as that health practices, approaches, knowledge and beliefs incorporating plant, animal and mineral based medicines, spiritual therapies, manual techniques and exercises, applied singularly or in combination to diagnose and treat fracture in human body.
In a further explanation, Ekere [6] reiterates that; traditional medicine is based on the belief that: the natural resources have active therapeutic principles that heal occult supernatural forces, power to change active principles which can be manipulated by those who know how to produce marvelous results. This implies that Africans have belief in using the natural way to treat illnesses than the modern and scientific method that was brought from the western societies [7].

In the main, superstition, ignorance and poverty are the basis for continued patronage despite complications [5]. There are however many complications attributed to the TBS. Indeed, the patrons of this service cuts across every strata of the society including the educated and the rich [3]. Mostly, Africans do believe that diseases and accidents have spiritual components that need to be tackled along with treatment. The major and commonest problems treated by them are fractures and dislocations [3].

\section{Problem}

In the main, before the emergence of western invention, every society stipulated methods of doing things even with relation to health. These methods are engrained in the culture and tradition of the people. As it is done in every other aspect, there are traditional means of treating the sick whenever there is the need to do so. African traditional medicine (Alternative medicine) therefore has an important place in the healthcare delivery system among Africans, especially in rural southwest Nigeria. Being widely known as alternative medicine, traditional medicines are utilized informs of treatment and also serves as last resort where other forms of treatment have failed. 
According to Omololu, Ogunlade and Gopaldsani [7], traditional medicine has been the first port of call before western or orthodox medicine and a last resort when all orthodox efforts fail. From this assertion, it is indicated that African people firstly put traditional medicine into consideration, whenever they are to undergo any treatment before looking at western method.

On the whole, traditional healers were long practiced before orthodox medicine was introduced into the developing world. The first orthodox hospital was built in Nigeria (Lagos) in 1873. Before this, traditional medicine was the only available form of healthcare, [7]. The art of traditional medicine is so wide that different experts have emerged to have their own area of specialization [8]. There is therefore no disputing the fact that some aspects of trado-medical knowledge system is well structured and organized and has survived through generations to maintain harmony between body, mind and soul within its socio-cultural and religious context. However, different experts have emerged within their ranks including herbalists, bonesetters, psychiatrists, and traditional birth attendants among others [8].

The traditional bone setter is a lay practitioner of joints manipulation. He/she is the unqualified practitioner (in the western training) who takes up the practice of healing without having had any formal training in accepted medical procedures according to Agaja [9].

\section{Functionalism theory}

Functionalism sees society as an organic whole, with each of its parts working to maintain the others. This is similar to the way in which parts of the body work to maintain each other and the body as a whole.

To study the function of a social practice or institution is to analyze the contribution which that practices or institution makes to the continuation of the society as a whole. The best way to understand this is through organic analogy; to study a bodily organ, we need to show how it relates to the other part of the body.

Functionalist perspective on health and medicine was formulated largely by Talcott Parsons. He explained that a healthy population is essential to the society. Healthy people can perform the social roles that are necessary to keep the society function optimally. Illness, then, is dysfunctional as it prevents people from performing their social roles, at least temporarily. Thus, the traditional bonesetters play a vital role in the overall functioning of a society by making members who have fractures regain their health. If those who have fractures are not treated, just like the organic analogy as explained by the functionalist, it will hamper the continued existence of the society, as the role they are supposed to play toward the survival of the society were affected.

The practice of traditional bone settings have though existed for centuries, there has however been campaigns against its patronage, especially by the orthodox practitioners. Despite this campaign, it still survives till today. This indicated that the practice of traditional bone setting has an important role that it is playing in the society, to have continued to exist. It would have ceased to be in existence, if it has no role that it is played in the society.

The functionalists explained that the society comprises of structures with each part laying different role toward ensuring the continual survival of the society. They used the organic analogy to explain this whereby organism contains different part, with each part carrying out a particular function toward the sustenance of the organism.
Though the functionalist perspective identified the social institution bone setting as being vital toward the overall functioning of the society by making the members healthy, the failure to recognize that there may be better ways of managing fractures, due to their rigidity and maintaining status quo therefore led to the need to another theory to explain this study

\section{Modernization theory}

1. The modernist theorists on the other hand look at how society evolves from tradition to modernity. They explained that societies will join the - developed\| world when they do away with their traditions and adopt modernity as a way of life.

2. In constructing their accounts of development, they drew on the tradition-modernity distinction of classical sociologists. They placed most emphasis on norms and values that operate in these two types of society. They argued that the transition from the traditional to modernity depended on a prior change in the values, attitudes and norms of people.

3. They called for the total abandoning of the old form of doing things, for the adoption of the western ways.

To synthesize these theories, functionalism and modernization theory, have a common perspective on the importance of ensuring that people who have fractures are treated and continue to contribute their own quota toward having a functional society, they however have point of divergence. While functionalism explain the relevance of the traditional bonesetters toward the continual survival of the society and believes in the maintenance of status quo, modernization theory looks at how the traditional bonesetters have adopted modern form in carrying out treatment among patients. Modernity has made it easier for the traditional bone setters to adopt modern form in the treatment of fracture. This is important as it not only reduce the rate of complication, but also assist in ensuring that proper treatment are received by the patients, which will aid them in their health restoration and optimal functioning in the society.

Both the Traditional bonesetters and the western practitioners are practicing today in Nigeria. Until recently, the relationship that exists between these two kinds of practitioners can best be explained as being that of cat and mouse as the traditional bone setters'method of treatment was regarded as being fetish, primitive and not modern.

\section{Methodology}

This study was conducted within Ekiti State. Many communities where traditional bone-setters and their clients could be found were selected for the study.

The communities included in the study were

1. Ado-Ekiti,

2. Ikole-Ekiti,

3. Oye-Ekiti,

4. Ise-Ekiti,

5. Efon-Ekiti,

6. Omuo-Ekiti,

7. Ijero-Ekiti and

8. Ogotun-Ekiti.

Although the number of traditional bone setters that abound in these communities is not well documented due to lack of proper 
Page 3 of 5

record by the constituted authorities, it is widely accepted that traditional fracture care is utilized in the selected communities.

Thus, the purposive and snowball sampling techniques were used traditional bonesetter practitioners or patients who have fractures. The first bonesetter clinic was identified by the researcher and snowballing technique was used to identify subsequent respondents/interviewees

Although patients were not evenly distributed in the clinics identified, the available number of patients that a clinic can catered for within the period of the study was all selected.

Patients in the clinics range between three and twenty-two. Both quantitative and qualitative methods were used to collect the necessary data. For the quantitative research method, Two hundred and fifty (250) copies of questionnaires were administered among patients in Ten traditional bonesetter's clinics that were identified and visited out of which Two hundred forty-seven (247) copies were retrieved for analysis, while the in-depth interview was conducted with traditional bonesetter practitioners in the Ten clinics and Fifteen patients that were visited.

Quantitative data was analyzed using descriptive statistical tools like tables, percentages, frequency distributions, bar charts and pie-charts, while qualitative data was content analyzed.

\section{Ethical consideration}

The anonymity of all respondents was of paramount significance to the researcher. At no point during and after the study was any information given was being used in a manner that would make it feasible to identify the respondent. Participant was given the right to decline from the study at any stage should he/she found it uncomfortable to continue. The study did not cause any harm to the participants in the process of administering questionnaire and indepth interviews. The participation of the respondents in the study was entirely voluntary.

\section{Findings}

This section deals with the analysis and interpretation of data.

\begin{tabular}{|l|l|l|}
\hline Age years & Frequency & Percentage \% \\
\hline Male & 166 & 67.2 \\
Female & 81 & 32.8 \\
Total & 247 & 100 \\
\hline Sex & Frequency & Percentage \% \\
\hline$\leq 20$ & 13 & 5.3 \\
$21-30$ & 57 & 23.1 \\
$31-40$ & 114 & 46.2 \\
$41-50$ & 35 & 14.2 \\
51 yrs and above & 28 & 11.3 \\
Total & 247 & 100 \\
\hline Educational Level & Frequency & Percentage \% \\
\hline No formal schooling & 24 & 9.7 \\
Primary school. & 69 & 27.9 \\
Secondary school & 100 & 40.5 \\
Tertiary school & 54 & 21.9 \\
\hline
\end{tabular}

\begin{tabular}{|l|l|l|}
\hline Total & 247 & 100 \\
\hline Ethnic Group & Frequency & Percentage \% \\
\hline Yoruba & 184 & 74.5 \\
Igbo & 4 & 1.6 \\
Hausa & 24 & 9.7 \\
Others & 35 & 14.2 \\
Total & 247 & 100 \\
\hline Religion & Frequency & Percentage \% \\
\hline Christianity & 133 & 53.8 \\
Muslim & 109 & 44.1 \\
Traditional belief & 5 & 2.0 \\
Total & 247 & 100 \\
\hline
\end{tabular}

Table 1: Distribution of Respondents' Demographic Characteristics.

Table 1 depicts that; out of the total numbers of respondents (247), $166(66.4 \%)$ of them were males. Age ranged between 15 and 72 years (mean \pm SEM was $35.44 \pm 3.71) .184(74.5 \%)$ participants were Yoruba ethnic group while $63(25.5 \%)$ from other ethic groups. The distribution of participants according to their educational status was varying. The findings showed that few of the respondents were not educated. Education level found to be of no role in the preference of treatment selection. $53.8 \%$ of the participants that were Christians still preferred to be treated by TBSs while $44.1 \%$ from rural descent preferred to be treated by TBSs

\section{Reasons of preference of TBSs}

Figure 1 below indicated that; $38 \%$ of the participants from urban community preferred to be treated by TBSs while $48 \%$ from rural descent preferred to be treated by TBSs, $\mathrm{P}=0.021$. 192 participants out of 199 responded well to the questionnaire.

The reason for going to TBS was; in 71 participants (37\%) was their beliefs, in 27 participants (14.06\%) was due to the low cost of TBS, and in 27 participants $(14.06 \%$ ) was due to fear of plaster (Figure 1).

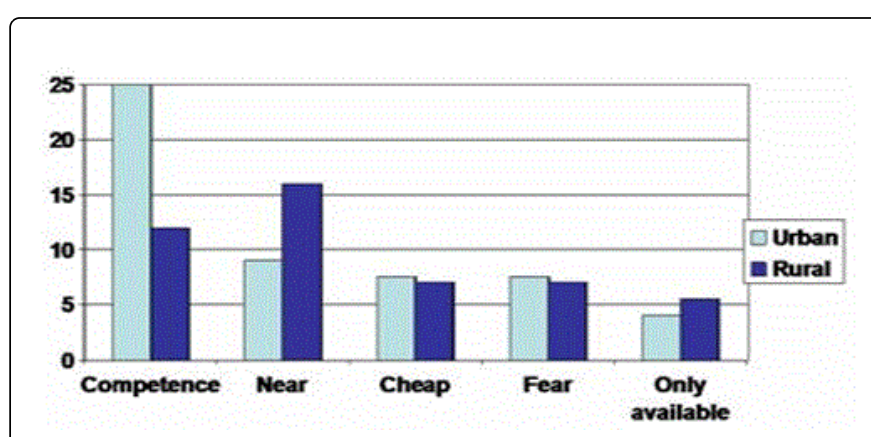

Figure 1: Participants From Urban Community and treatment with TBS's.

\section{TBSs utilization}

Examination of the TBSs patronage, 70 (28.3\%) of the clients had a past history of treatment by an orthodox practitioner in their previous condition, while 177 (71.7\%) were not but the first experience. On the 
whole, $124(50.2 \%)$ of clients were attended to by bone-setters immediately in their current condition, 123 (49.8\%) were not but still kept waiting.

Furthermore, many of those who had been attended to by the orthopaedists in the hospital and still fell back to TBSs indicated various reasons for doing so. The reasons of why do they changed to TBSs were varied. Out of the total respondents, 23 (9.3\%) of the clients indicated that, the TBS is the only available nearby service to be utilized, 224 (90.7\%) portrayed different medical causes for patronizing TBSs, which ranged from delay in medical services in the hospitals, fear of cast or amputation, occupied wards, less trained doctors, and high cost of hospital treatment $(11.2 \%, 30.8 \%, 17.7 \%$, $9.1 \%, 9.7 \%$, respectively), and in $15(11.5 \%)$ because of their beliefs (Figure 2).

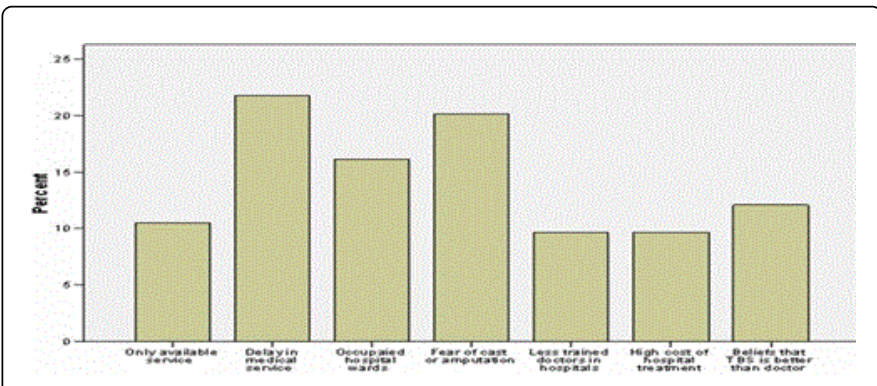

Figure 2: Examination of the TBSs patronage.

This section depicts the end result of the treatment of fracture through TBSs

\begin{tabular}{|l|l|l|}
\hline Out come & Frequency & $\mathbf{( \% )}$ \\
\hline Poor & 7 & $(2.8)$ \\
Acceptable & 33 & $(13.4)$ \\
Good & 84 & $(34.0)$ \\
Excellent & 123 & $(49.8)$ \\
\hline Total & 247 & $(100.0)$ \\
\hline
\end{tabular}

Table 2: Outcome of Treatment Received By the Clients through TBSs.

In the main, investigation about the outcome of their treatment; majority $(49.8 \%)$ of the respondents indicated that it was excellent and poor in $7(2.8 \%)$ (Table 2). This result really shows the strength of the TBSs in fracture care in Ekiti State, especially the rural communities (Figure 3).

The Figure 3 above is an investigation on the decision maker on utilization of TBSs by the clients. The decision to contact the TBSs was a self-decision in $68(27.5 \%)$, family in $99(40.1 \%)$, relatives in 61 $(24.7 \%)$, and friend decision in $19(7.7 \%)$.

\section{Source of knowledge of TBSs}

An investigation on the source of their knowledge in bone setting, it was revealed that majority of the TBSs interviewed got into the system from self-education, parents or from relatives, and many others indicated that they got into the practice through other different sources.

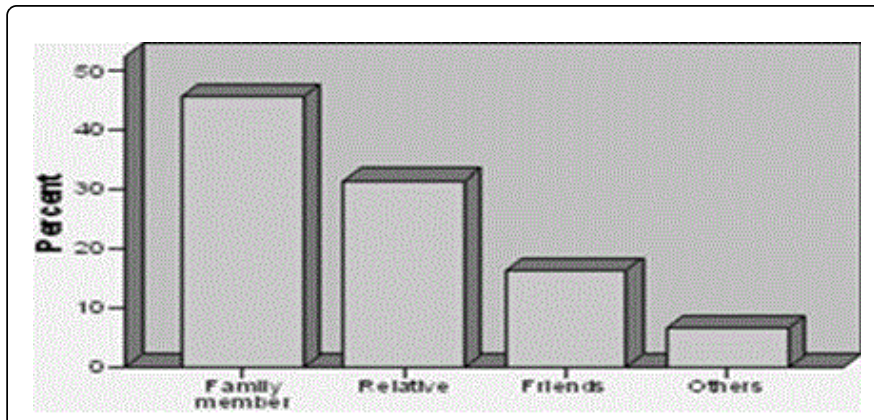

Figure 3: Decision on Service Utilization.

\section{Discussion}

The study revealed that males (67.2\%) accounted for a large portion of patients seeking TBS treatment and showing that males are predominantly injured. This is no other reason than injuries from accident on "Okada" (Motorcycles). Majority of the unemployed graduates in Ekiti are daily engaging in using motorcycles for commercial purpose in a bid to make ends meet.

Hundred twenty-four (124) of the TBS's clients (50.2\%) went to TBS from the start of their injury, this result agrees with Owumi et al. study, where $74.9 \%$ of patients were seen initially by TBS [8]. This could be dangerous especially in those that could have sustained concomitant life threatening injuries.

In the main, it is interesting that $49.8 \%$ of the patients initially were taken to hospitals before being withdrawn and attended treatment by TBS. This result is comparable to studies by Olorun [10] and Solagberu [11], where the incidences were $50 \%$ and $43 \%$ respectively. This high percentage requires an audit of our orthodox system in order to correct over inadequacies.

Different kind of fractures and dislocation were managed by the TBS in this study using the splints, herbs, skin lashes, pads and manipulation without consideration for a perfect anatomical reduction and alignment. It is important to raise the level of awareness of our communities about the narrow limit of the TBS in making a diagnosis and proper treatment of orthopaedic and trauma problems and this only can be tackled through a proper health education programs.

The reasons for continued patronage or preference and the attitude of the people and the TBSs are similar in studies conducted in Africa [12].

Patronage as shown in this study is determined by several factors. These factors were found not to be restricted to a particular group of people but involve people from every social strata and academic classes. Findings from the study also indicated that the TBS treatment is also believed to be cheap to the clients.

On the whole, fear of amputation was the reason of patronage in a small percentage of this study (17.7\%), this closely agrees with Ogunlusi's [2] study who found that the fear of amputation was in $7 \%$. It is important to educate people and let them know that Modern Orthopaedic Services does primarily save the limbs, and amputation is carried out on limbs that cannot be saved or for dead ones. We revealed that one of the important preference determinant factors included the opinions of family and friends. We found that the decision of seeking treatment at TBS in $68.1 \%$ of clients is non-self- 
Citation: Sina OJ, Ayodele IM (2015) Traditional Bone-Setters and Fracture Care in Ekiti State, Nigeria. Altern Integr Med 4: 182. doi: $10.4172 / 2327-5162.1000182$

Page 5 of 5

decision (family members and friends); this closely agrees with Solagberu and Dada et al, where they found that the initial idea of visiting TBS was from an external person in $75 \%$ and 25 of cases respectively $[10,11]$.

\section{Conclusion and Recommendations}

In view of the societal confidence, which the TBS enjoy in Nigeria, it is important that efforts be made at regulating their practice including the establishment of a sound referral system and adoption of a standard training curriculum. Though a number of deficiencies of the bone setters have been highlighted in this paper, it is obvious that they can be trained to function at the primary level especially in the rural areas.

1. Although there is a number of qualified orthopaedic surgeons and well-equipped hospitals in Nigeria at the moment, treatment is still obtained from traditional bonesetters by a large number of our population.

2. In spite of the complications, TBS continue to have patronage from both the highly educated and the illiterate. Possible reasons for this include culture and beliefs, ignorance and third-party advice, and also overcrowding of hospitals with trauma cases.

3. More collaborative effort is needed to raise awareness of complications that may arise from seeking management in TBS.

4. To achieve that, an extensive study of the current status of TBS including the obstacles and motivations for change is mandatory.

\section{References}

1. Orjioke CJG (2005) Does traditional medicine have a place in Primary Health Care? Orient J of Medicine.
2. Ogunlusi DO, Ikem IC, Oginni (2007) Why Patients Patronize Traditional Bone Setters. The Internet Journal of Orthopedic Surgery 4: 2 .

3. Thanni LO (2000) Factors influencing patronage of traditional bone setters. West Afr J Med 19: 220-224.

4. Oyebola DD (1980) Yoruba traditional bonesetters: the practice of orthopaedics in a primitive setting in Nigeria. J Trauma 20:312-322.

5. Udosen AM, Etiuma AU, Ugare GA (2005) Femoral Artery Aneurysm, a Complication of Traditional Bonesetting, (A case report). NigJSurg.

6. Ekere AU (2003) The scope of extremity amputations in a private hospital in the south-south region of Nigeria. Niger J Med 12: 225-228.

7. Omololu AB, Ogunlade SO, Gopaldasani VK (2008) The practice of traditional bonesetting: training algorithm. Clin Orthop Relat Res 466: 2392-2398.

8. Owumi BE, Jerome T (2012) Yoruba traditional bonesetters: the practice of orthopaedics in a primitive setting in Nigeria. J trauma 20: 312-322.

9. Agaja SB (2000) Prevalence of limb amputation and role of TBS in Nigeria. Nig J surgery 7: 79.

10. OlaOlorun DA, Oladiran IO, Adeniran A (2001) Complications of fracture treatment by traditional bonesetters in southwest Nigeria. Fam Pract 18: 635-637.

11. Solagberu BA (2005) Long bone fractures treated by traditional bonesetters: a study of patients' behaviour. Trop Doct 35: 106-108.

12. Ikpeme IA, Udosen AM, Okereke-Okpa I (2007) Patients' perception of traditional bone setting in Calabar. Port Harcourt medical Journal 1: 104-108. 